\title{
Lactobacillus supplementation for diarrhoea related to chemotherapy of colorectal cancer: a randomised study
}

\author{
P Österlund", , T Ruotsalainen', R Korpela ${ }^{2,3}$, M Saxelin ${ }^{3}$, A Ollus ${ }^{4}$, P Valta ${ }^{5}$, M Kouri', I Elomaa' and \\ H Joensuu'
}

'Department of Oncology, Helsinki University Central Hospital, PO Box 180, 00029 HUS Helsinki, Finland; ${ }^{2}$ Institute of Biomedicine, University of Helsinki, PO Box 63, Fl-000 4 Finland; ${ }^{3}$ Valio Ltd, R\&D, PO Box 30, Fl-00039 VALIO, Helsinki, Finland; ${ }^{4}$ Department of Internal Medicine, Helsinki University Central Hospital, PO Box 340, Fl-00029 Helsinki, Finland; '5epartment of Anaesthesiology, Helsinki University Central HospitallJorvi Hospital, Turuntie 150, Fl-02740 Espoo, Finland

5-Fluorouracil (5-FU)-based chemotherapy is frequently associated with diarrhoea. We compared two 5-FU-based regimens and the effect of Lactobacillus and fibre supplementation on treatment tolerability. Patients diagnosed with colorectal cancer $(n=150)$ were randomly allocated to receive monthly 5-FU and leucovorin bolus injections (the Mayo regimen) or a bimonthly 5-FU bolus plus continuous infusion (the simplified de Gramont regimen) for 24 weeks as postoperative adjuvant therapy. On the basis of random allocation, the study participants did or did not receive Lactobacillus rhamnosus GG supplementation ( $1-2 \times 10^{10}$ per day) and fibre ( I I g guar gum per day) during chemotherapy. Patients who received Lactobacillus had less grade 3 or 4 diarrhoea (22 vs 37\%, $P=0.027$ ), reported less abdominal discomfort, needed less hospital care and had fewer chemotherapy dose reductions due to bowel toxicity. No Lactobacillus-related toxicity was detected. Guar gum supplementation had no influence on chemotherapy tolerability. The simplified de Gramont regimen was associated with fewer grade 3 or 4 adverse effects than the Mayo regimen (45 vs $89 \%$ ), and with less diarrhoea. We conclude that Lactobacillus GG supplementation is well tolerated and may reduce the frequency of severe diarrhoea and abdominal discomfort related to 5-FU-based chemotherapy.

British Journal of Cancer (2007) 97, 1028 - 1034. doi:I0.1038/sj.bjc.6603990 www.bjcancer.com

Published online 25 September 2007

(c) 2007 Cancer Research UK

Keywords: chemotherapy; colorectal cancer; 5-fluorouracil; Lactobacillus rhamnosus GG; probiotic

Colorectal cancer is globally the third most common type of cancer and the fourth most common cause of cancer death (Parkin et al, 1999; Pisani et al, 1999). Curative surgery is feasible in threequarters of the patients, but despite this, about one half of the patients subsequently develop incurable recurrent cancer (Galandiuk et al, 1992). Adjuvant chemotherapy or chemoradiation reduces recurrences and mortality in colorectal cancer (Van Cutsem et al, 2002). Regimens containing 5-fluorouracil (5-FU) and leucovorin (LV) have been considered as standard adjuvant chemotherapy regimens in colorectal cancer (O'Connell et al, 1998; Wolmark et al, 1999; Kerr, 2001), and addition of oxaliplatin to 5-FU and LV appears to further improve efficacy (Andre et al, 2004).

Diarrhoea is one of the most troublesome adverse effects related to cancer chemotherapy. 5-Fluorouracil-, capecitabine-, and irinotecan-based regimens that are commonly used in the treatment of colorectal cancer are frequently associated with diarrhoea. Excessive bowel motility may be reduced using drugs such as loperamide and somatostatin analogues, but these treatments may also be associated with adverse effects, and simple and safe measures to reduce drug-induced diarrhoea are thus needed. The mode of chemotherapy administration may also

*Correspondence: Dr P Österlund; E-mail: pia.osterlund@hus.fi Received 30 May 2007; revised 23 July 2007; accepted I4 August 2007; published online 25 September 2007 influence chemotherapy-related toxicity. Regimens where 5-FU is administered as protracted continuous infusions may result in a more favourable toxicity profile including the frequency and severity of diarrhoea as compared to the Mayo regimen, where 5 -FU is given as boluses on 5 consecutive days 4-weekly (de Gramont et al, 1997a).

According to a meta-analysis of controlled trials performed on hospitalised children who have acute diarrhoea, co-administration of some microorganisms (probiotics) such as Lactobacillus rhamnosus GG with standard rehydration therapy reduces the duration of diarrhoea (Huang et al, 2002). Some placebocontrolled studies also suggest that probiotics are of benefit in the treatment of antibiotics-associated diarrhoea and in the prevention of nosocomial diarrhoea in infants (Szajewska et al, 2001; Cremonini et al, 2002). The putative mechanisms of L. rhamnosus GG action may include stimulation of the cell proliferation rate of bowel epithelial cells, enhanced secretion of protective mucins leading to reduced adherence of enteropathogenic bacteria to the bowel wall, inhibition of bacterial translocation into the tissues, and stimulation of local and systemic immune response to pathogens (Mattar et al, 2001; Banasaz et al, 2002; Khaled et al, 2003; Mack et al, 2003; Vaarala, 2003). Partially hydrolysed guar gum fibre may also reduce duration of diarrhoea (Homann et al, 1994; Alam et al, 2000) and prolong the colonic transit time (Meier et al, 1993). Thus, hypothetically, besides the 
mode of 5-FU administration, the frequency and severity of chemotherapy-associated gastrointestinal adverse events might be influenced by the diet and the bowel microbial flora.

Three studies have suggested that Lactobacillus acidophilus, $L$. rhamnosus, or a probiotic mixture may prevent radiotherapyinduced diarrhoea (Salminen et al, 1988; Urbancsek et al, 2001; Delia et al, 2002), but to our knowledge no controlled study has evaluated probiotics or fibre in the prevention of chemotherapyassociated diarrhoea. In the present study, we assessed the efficacy of L. rhamnosus GG and guar gum supplementation in reducing 5-FU-based chemotherapy toxicity. We also compared the tolerability and the frequency of diarrhoea related to the Mayo regimen to that of the simplified de Gramont regimen, which uses a bolus plus continuous 5-FU infusion.

\section{METHODS}

\section{Study design and accrual}

The primary end point of this open-label, prospective, randomised, phase III, single institution, $2 \times 3$ factorial design study was the frequency of severe diarrhoea. The study participants had either Dukes' B or C colorectal cancer $(n=126)$ or metastatic colorectal cancer that had been rendered free from all overt metastases by surgery (Dukes' $\mathrm{D}, n=24$ ). All patients received adjuvant chemotherapy following surgery. Chemotherapy consisted either of the Mayo regimen or the simplified de Gramont regimen, and was administered based on random allocation. In addition, study participants diagnosed with rectal cancer received locoregional radiotherapy whenever the caudal tumour margin was below the distal peritoneal fold.

One hundred and fifty-four subjects were assessed for the study between November 1997 and August 2001. Of these, one was ineligible due to age and three others preferred not to participate leaving a total of 150 eligible patients who consented to participate in the study. An Institutional Review Board at Helsinki University Central Hospital approved the study protocol prior to initiation of the study. A written informed consent was required from the participants prior to study entry.

\section{Treatment assignment}

Allocation to the study treatments was performed using a computerised minimisation technique (Pocock and Simon, 1975; Freedman and White, 1976) and one out of six chances. The patients were randomly allocated at a $1: 1$ ratio to receive either the simplified de Gramont regimen or the Mayo regimen as adjuvant chemotherapy. The participants were also randomly assigned to receive or not to receive at a $2: 1$ ratio $L$. rhamnosus GG and at a $1: 2$ ratio fibre-containing nutritional support (guar gum). The allocation group was concealed until interventions had been assigned. The patients were stratified by gender, tumour site (colon or rectum), and the Dukes' stage at randomisation.

\section{Participant eligibility}

Subjects were eligible for inclusion provided that age at randomisation was 18 or higher and 75 or lower, histologically confirmed colorectal cancer had been removed at surgery, no metastases were found in staging examinations that included ultrasound or computed tomography (CT) of the abdomen and chest $\mathrm{X}$ ray or CT of the thorax, and the World Health Organization (WHO) performance status was two or less. The study participants were also required to have adequate bone marrow, kidney, and liver functions for chemotherapy. Exclusion criteria included other invasive cancer beside colorectal cancer in history except for carcinoma in situ of the cervix or nonmelanoma skin cancer; metabolic, neurological, or psychiatric disease that was incompatible with chemotherapy; a serious thromboembolic event under active treatment; and pregnancy, lactation, or absence of adequate contraception in potentially fertile patients.

\section{Adjuvant systemic chemotherapy and radiation therapy}

The Mayo regimen consisted of a short intravenous infusion of $\mathrm{LV} 20 \mathrm{mg} \mathrm{m}^{-2}$ (or $10 \mathrm{mg} \mathrm{m}^{-2}$ levoleucovorin) and $5-\mathrm{FU}$ $370-425 \mathrm{mg} \mathrm{m}^{-2}$ administered as an intravenous bolus over $3-5$ min on days $1-5$ of the cycle, which was repeated at 4-week intervals for six times. The overall duration of chemotherapy was thus 24 weeks.

The simplified de Gramont regimen consisted of a 2-h infusion of $\mathrm{LV} 400 \mathrm{mg} \mathrm{m}^{-2}$ (or $200 \mathrm{mg} \mathrm{m}^{-2}$ levoleucovorin) followed by 5-FU $400 \mathrm{mg} \mathrm{m}^{-2}$ administered as an intravenous bolus and $48-\mathrm{h}$ infusion of $3.0-3.6 \mathrm{~g} \mathrm{~m}^{-2} 5$-FU; this cycle was repeated every 14 days for 12 times. The overall duration of chemotherapy was thus 24 weeks (de Gramont et al, 1997b).

Pelvic radiation therapy for rectal cancer was given from three or four portals using high-energy photons obtained from a linear accelerator $(n=39)$. Radiotherapy, based on CT planning, was administered to a total cumulative dose of $50.4 \mathrm{~Gy}$ in $1.8 \mathrm{~Gy}$ daily fractions over 5.5 weeks except for patients who underwent abdominoperineal resection, when the dose was limited to $45 \mathrm{~Gy}$ to decrease the likelihood of small bowel radiation injury $(n=8)$. Leucovorin was omitted during pelvic radiotherapy (cycles three and four) in rectal cancer patients assigned to receive the Mayo regimen, and bolus 5-FU, $500 \mathrm{mg} \mathrm{m}^{-2}$, was given only for 3 days during these cycles. Similarly, rectal cancer patients assigned to receive the simplified de Gramont regimen did not receive LV during pelvic radiotherapy (cycles five to eight) and were treated with continuous 5-FU infusion alone, $225 \mathrm{mg} \mathrm{m}^{-2}$ per day (O'Connell et al, 1994). Seven rectal cancer patients were treated to a cumulative target dose of $25 \mathrm{~Gy}$ given as five equal fractions over 5 consecutive days preoperatively (Anonymous, 1996; Kapiteijn et al, 2001); no concomitant 5-FU was given to these patients during radiation, and their postoperative systemic chemotherapy was given as for colon cancer patients.

\section{Dietary supplementation}

Lactobacillus rhamnosus GG (ATCC 53103, Gefilus ${ }^{\circledR}$, Valio Ltd, Helsinki, Finland) was administered orally as gelatine capsules twice daily at a dose of $1-2 \times 10^{10}$ per day during the 24 weeks of adjuvant cancer chemotherapy. Lactobacillus capsules were swallowed as such, or the capsule content was dissolved in cold milk or juice. Guar gum containing nutritional supplement $\left(500 \mathrm{ml}\right.$, Novasource GI control ${ }^{\mathrm{R}}$, Novartis Nutrition, Basel, Switzerland (contains $11 \mathrm{~g}$ guar gum and $550 \mathrm{kcal}$ or $2300 \mathrm{~kJ}$ ), was administered daily, on cycle days $7-14$, for 8 days per month. All patients received dietary counselling.

\section{Concomitant medications}

No other dietary supplements were allowed during the study. Treatment compliance was monitored using nutrition diaries. Prophylactic antibiotics or leukocyte growth factors were not routinely prescribed during chemotherapy, but the patients were allowed to use any medication deemed necessary for appropriate care of concomitant diseases. Metoclopramide and 5-HT3 inhibitors were used for nausea/vomiting, loperamide for diarrhoea, dexpanthenol lozenges 100-200 mg t.i.d. for stomatitis, and pyridoxine $50 \mathrm{mg}$ t.i.d. for hand-foot syndrome at the discretion of the treating physician. 


\section{Monitoring of treatment-related adverse effects}

Patients were scheduled to be evaluated within 21 days prior to study treatment initiation, 4 weekly during chemo- and radiotherapy, and at protocol-determined intervals (ranging from 2 to 6 months) posttreatment. At each visit, medical history was taken and physical examination was performed, which included the WHO performance status and weight, and the blood cell counts and serum chemistry were analysed. Blood cell counts were monitored at 10 to 14-day intervals. Treatment-related adverse effects were evaluated at every cycle using a diary kept by the patients and by a physician (PÖ or TR). Adverse events were assessed and graded according to the Common Toxicity Criteria of the National Cancer Institute of Canada scale version 2.

\section{Number of patients, power assumptions, and statistical methods}

No reliable estimation of L. rhamnosus GG efficacy in reduction of chemotherapy-associated diarrhoea could be made prior to the study due to lack of relevant data. The frequency of grades 3 and 4 diarrhoea was the primary variable. Assuming a $50 \%$ reduction in the frequency of grade 3 or 4 diarrhoea from $40-50 \%$ to $20-25 \%$, using 2: 1 allocation between the study arms, an $80 \%$ power, and a 0.05 significance level, approximately 150 patients need to be accrued to the study. On the basis of prior data (de Gramont et al, 1997a), patients who receive continuous 5-FU might have less adverse effects than those treated with bolus 5-FU. A two-group $\chi^{2}$ test at the 0.05 significance level (two-sided) has an $80 \%$ power to detect a difference in the frequency of diarrhoea between the proportions of 0.60 or 0.40 in the bolus regimen group and proportions of 0.35 or 0.20 in the continuous 5-FU administration group, when the respective sample sizes are 62 and 82, also suggesting that accrual of approximately 150 patients allows detection of approximately $20 \%$ difference in the rate of diarrhoea between the groups. No interactions between chemotherapy and nutritional supplements were assumed.

The study was analysed according to the intention-to-treat principle, and outcome was analysed as defined in the study protocol. The statistical analyses were performed with a StatView computer program (SAS institute, Abacus concepts incorporation, Berkeley, CA, USA). The effects of bolus 5-FU vs continuous 5-FU regimens, chemoradiation $v s$ chemotherapy only, L. rhamnosus GG $v s$ no dietary supplements, and guar gum $v s$ no guar gum were compared using univariate and multivariate logistic regression models. The results are given as odds ratios with $95 \%$ confidence intervals. Mann-Whitney $U$-test was used to compare the treatment groups with respect to quantitative response variables. Frequency tables were analysed using the $\chi^{2}$ test.

\section{RESULTS}

\section{Patient characteristics and compliance}

The treatment arms were balanced with gender, the WHO performance status, primary tumour site, Dukes' stage, and radiation therapy given (Table 1 ). The median age at randomisation was 60 (range: $31-75$ ); $51 \%$ of the participants were men. Sixteen $(11 \%)$ subjects did not complete the scheduled 6 months of adjuvant chemotherapy due to either adverse events $(n=7$, six of whom received bolus 5-FU), cancer recurrence $(n=5)$, or a concomitant disease $(n=4)$. Two patients (both in the continuous 5-FU group) who did not receive any of the study treatments due to postoperative complications were not included in safety or efficacy analyses leaving 148 patients for these analyses. None of the patients were lost to follow-up.

\section{Chemotherapy dose intensity and tolerability}

The scheduled 5-FU dose intensity, calculated as the percentage of the scheduled dose as milligrams of 5-FU given per square metre per week of the scheduled cumulative dose, was maintained better among the patients who received the simplified de Gramont regimen than among those treated with the Mayo regimen (median 93 vs $78 \%$, respectively; $P<0.0001$ ).

The simplified de Gramont regimen was tolerated better than the Mayo regimen (Table 2). Any grade 3 or 4 adverse effect was present in $87 \%$ ( 65 out of 75 ) of the patients treated with the Mayo regimen as compared with only $45 \%$ (33 out of 73 ) of those treated with the simplified de Gramont regimen, when the vascular access device (VAD)-related toxicity was included in this analysis $(P<0.0001$, Table 2$)$. Vascular access device-related complications occurred in seven $(10 \%)$ patients who received continuous $5-\mathrm{FU}$ infusions; three were classified as serious. The Mayo regimen was

Table I Patient characteristics

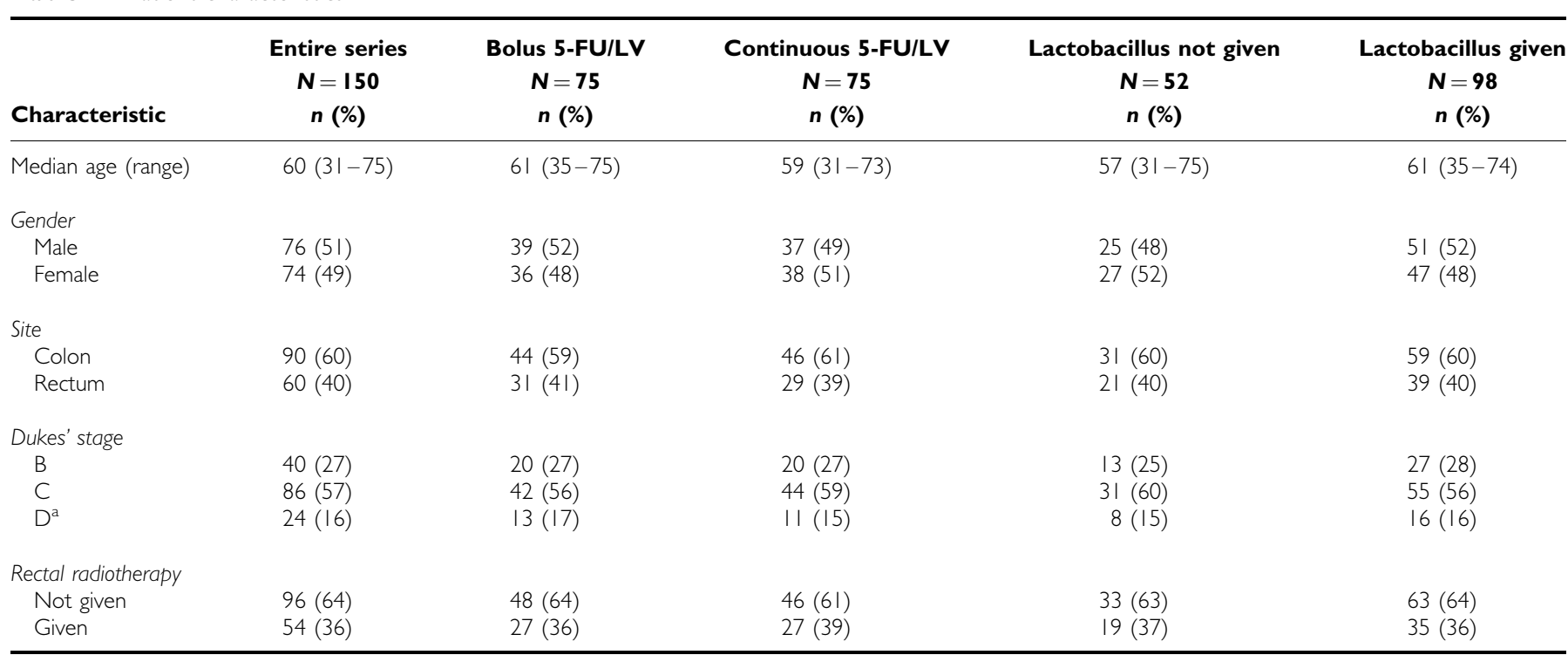

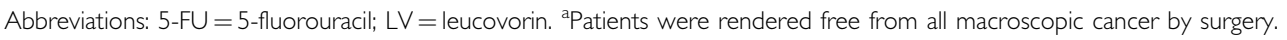


Table 2 Treatment-related adverse effects

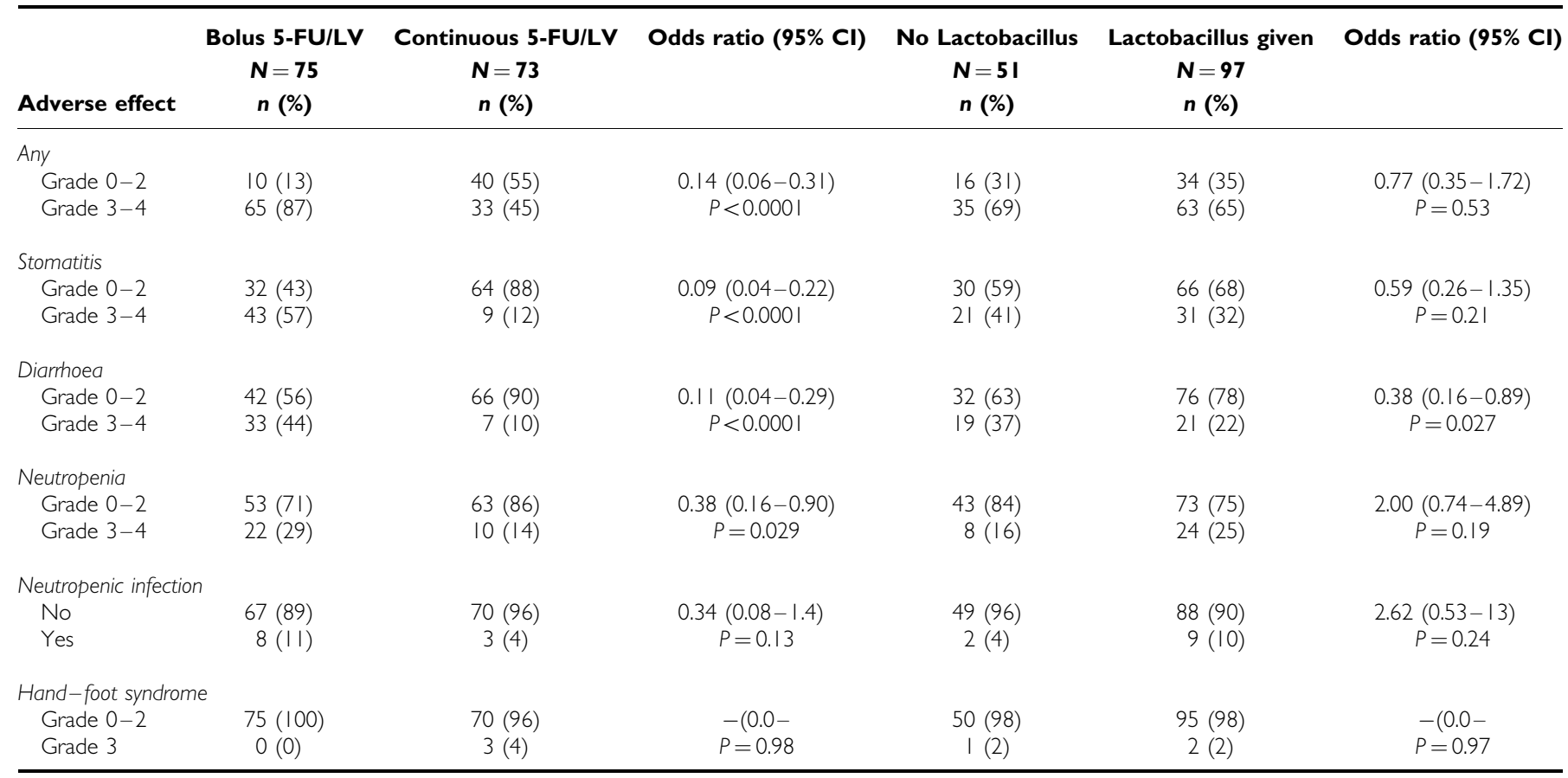

Abbreviations: $\mathrm{Cl}=$ confidence interval; 5-FU = 5-fluorouracil; $\mathrm{LV}=$ leucovorin.

more frequently associated with stomatitis, diarrhoea, and neutropenia than the simplified de Gramont regimen, whereas mild-to-moderate hand-foot syndrome was more common among the patients who received the simplified de Gramont regimen $(P<0.0001)$. There were no treatment-related deaths.

\section{Lactobacillus supplementation}

Forty-nine (51\%) of the 97 patients randomly allocated to receive oral Lactobacillus supplementation and $26(51 \%)$ of the 51 patients who were allocated to the control group were treated with the Mayo regimen $(P=0.96)$. Compliance to Lactobacillus supplementation was excellent, and all patients consumed their scheduled doses. The frequency of grade 3-4 diarrhoea was lower among patients who received Lactobacillus supplements than in the rest of the patients (22 vs 37\%; $P=0.027$, Figure 1). Seventy-six $(78 \%)$ patients in the Lactobacillus supplementation group and 43 (84\%) in the control group reported diarrhoea of any grade during the study (tested grade $0 v s>0, P=0.39$ ). Abdominal discomfort resulting from flatulence, borborygmia, or abdominal distension was less in patients who received Lactobacillus supplements (grade 2 or 3 in 2 vs 12\%, $P=0.025)$, and any grade of abdominal discomfort was present in $57(59 \%)$ of the patients who received Lactobacillus versus in $38(75 \%)$ of those who did not $(P=0.058)$. However, Lactobacillus supplementation had no significant effect on the overall toxicity of treatment, or the frequency of stomatitis or neutropenia (Table 2).

None of the patients had Lactobacillus GG growth in blood bacterial cultures. Nine (10\%) patients allocated to receive Lactobacillus had neutropenic infection as compared to two (4\%) of those who did not receive it $(P=0.24)$. Eight patients $(8 \%)$ in the Lactobacillus group required hospital care for bowel toxicity, as compared to $11(22 \%)$ in the comparator group $(P=0.021)$. Twenty (21\%) of the patients who received Lactobacillus supplementation had chemotherapy-dose reductions due to bowel toxicity as compared to $24(47 \%)$ among those who did not receive Lactobacillus $(P=0.0008)$. Only one patient discontinued

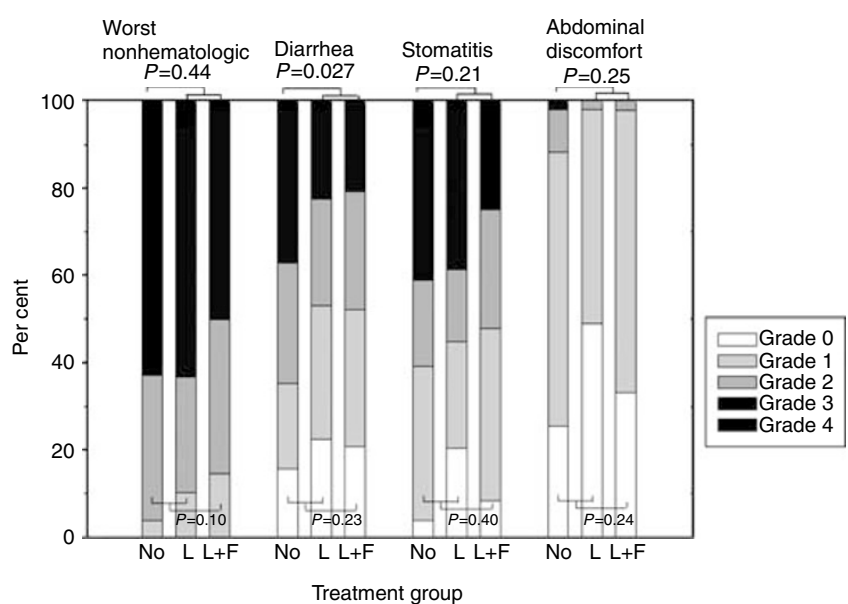

Figure I Effect of oral Lactobacillus rhamnosus GG (L) and Lactobacillus rhamnosus GG plus fibre (guar gum, L+F) supplementation on adverse events recorded during 5-FU-based chemotherapy.

chemotherapy primarily due to bowel toxicity (this patient was allocated not to receive Lactobacillus supplementation).

\section{Fibre supplementation}

Nine (18\%) patients discontinued fibre supplementation due to a taste aversion (these patients were included in the analysis according to the intention-to-treat principle). Addition of fibre did not influence the overall gastrointestinal toxicity $(P=0.13)$. Guar gum supplementation did not reduce the frequency of severe diarrhoea as compared to patients who did not receive fibre (25 vs $30 \%$, respectively; $P=0.24$ ). There was no difference between the allocation groups in the proportions of patients who had abdominal discomfort resulting from flatulence, borborygmia, or abdominal distension (grade 2 or 3 in $2 v s 7 \%, P=0.24$ ), and the 
rates of chemotherapy-dose reductions due to bowel toxicity were also similar between the groups $(P=0.20)$.

\section{DISCUSSION}

Chemotherapy-related diarrhoea is a common adverse effect in the treatment of colorectal cancer, since for example 5-FU, capecitabine, and irinotecan administration is frequently associated with diarrhoea. Severe diarrhoea may lead to nutritional and metabolic imbalances, and severe neutropenia associated with diarrhoea may be life threatening. The present findings indicate that the frequency of grade 3 or 4 diarrhoea may be reduced with the use of Lactobacillus supplementation. The latter finding is of interest, since Lactobacillus supplementation appears to have few or no adverse effects, Lactobacillus capsules are simple to administer, and they are associated with low costs. Patients who received Lactobacillus during chemotherapy reported less abdominal discomfort than those who did not receive it, and these subjects had also fewer chemotherapy-dose reductions, which might have an impact on chemotherapy efficacy. As many other bacteria, lactobacilli may occasionally cause septicaemia in severely immunocompromised patients (Salminen et al, 2004), but $L$. rhamnosus was identified in none of the blood cultures during the study. There was no difference between the allocation groups in the frequency of neutropenic fever.

Somewhat unexpectedly, nutritional supplements have not been evaluated in the prevention and treatment of chemotherapyrelated gastrointestinal adverse effects in controlled studies. In one study, dosing of Lactobacillus plantarum during 5-FU administration improved food intake and helped to maintain the body weight in rats, but it did not prevent diarrhoea (Von Bultzingslowen et al, 2003). Instead, probiotics have been studied in a variety of bowel diseases other than cancer in humans. Lactobacillus rhamnosus GG has been found to alleviate diarrhoea caused by a viral infection or Clostridium difficile, and to be useful in the prevention of traveller's diarrhoea or diarrhoea related to administration of antibiotics (Ouwehand et al, 2002; Vaarala, 2003). Probiotics may also reduce radiation therapy-related diarrhoea (Salminen et al, 1988; Urbancsek et al, 2001; Delia et al, 2002). The mode of action is not fully understood, but probiotics are involved in some cytoprotective processes, such as induction of heat-shock protein expression in intestinal epithelial cells (Tao et al, 2006), and prevention of cytokine-induced epithelial cell damage (Yan et al, 2007).

The combination of hydrolysed guar gum fibre and lactobacilli has been suggested to be effective for diarrhoea (Meier et al, 2003). One-third of the present patients received this combination, but we detected no further reduction in gastrointestinal adverse effects among patients who received the combination. The optimal dose and schedule to administer fibre are not known. We administered $11 \mathrm{~g}$ hydrolysed guar gum fibre daily for 8 days per month, but this dose may have been too low or the intervention duration too short. We chose not to administer guar gum concomitantly with chemotherapy to avoid nausea, because some individuals find its taste aversive.

The simplified de Gramont regimen that includes 5-FU given both as a bolus and as a 48-h continuous infusion was found to be better tolerated than the Mayo regimen, where 5-FU is given as boluses only. Earlier comparisons involving continuous and protracted 5-FU regimens are in line with present findings suggesting that the more protracted regimens are generally associated with less adverse effects (de Gramont et al, 1997a; Andre et al, 2003; Kohne et al, 2003; Saini et al, 2003). Efficacy comparisons between the de Gramont regimen or its modifications and the Mayo regimen suggest that the former are no less effective (de Gramont et al, 1997a; Andre et al, 2003).

Grade 3 or 4 adverse effects were frequently reported in the present series; $45 \%$ of the patients treated with the simplified de Gramont regimen and $87 \%$ of those treated with the Mayo regimen had at least one grade 3 or 4 adverse effect. These figures are higher (Andre et al, 2003) or approximately similar in frequency (Labianca et al, 1995) as those reported from studies where similar types of chemotherapy have been administered in the adjuvant setting. Use of radiation therapy in rectal cancer, and the patients keeping a diary may have increased the number of reported events in the present series. We administered 5-FU as true boluses with an approximate injection time of $3 \mathrm{~min}$, which may be associated with more adverse events than short (about $15 \mathrm{~min}$ ) infusions (Andre et al, 2001). We also included VAD-related adverse effects, though they were infrequent in comparison to some other series (Carde et al, 1989; Gleeson et al, 1993; Kock et al, 1998).

The chemotherapy regimens investigated did not contain irinotecan, capecitabine, or oxaliplatin that are now commonly used to treat colorectal cancer, which is a limitation of the study. The study was not placebo-controlled nor blinded to administration of the dietary supplements, which may or may not have influenced assessment of adverse effects. To remedy these potential shortcomings, we have initiated a prospective, randomised, multicentre, double blind, placebo-controlled study with a crossover design where we investigate the effects of Lactobacillus supplementation in conjunction with chemotherapy that contains capecitabine, oxaliplatin, irinotecan, and bevacizumab.

We conclude that daily oral administration of L. rhamnosus GG may reduce the frequency of severe 5-FU-based chemotherapyrelated diarrhoea, whereas fibre supplementation may be of little benefit. Lactobacillus supplementation may be a practical and welltolerated means to reduce the severity of 5-FU-based chemotherapy-induced diarrhoea, and deserves to be evaluated further.

\section{COMPETING INTERESTS}

TR, AO, PV, MK, IE, and HJ declare no conflict of interest. PÖ has received an honorarium from Baxter for teaching. MS is an employee at Valio Research center, Valio Ltd., Finland. RK is an employee at Valio Research center and University of Helsinki, Institute of Biomedical Sciences. This is an investigator-initiated and conducted study. Lactobacillus rhamnosus GG capsules were provided free-of-charge for the study by Valio. Novasource GI control and infusing devices were purchased from Novartis Finland and Baxter Finland at a discounted price for the study. The investigators collected all study data, had access to all data, and wrote the manuscript.

\section{ACKNOWLEDGEMENTS}

We acknowledge the Cancer Society of Finland and the Finnish Medical Association (Finska Läkaresällskapet) for financial support.

\section{REFERENCES}

Alam NH, Meier R, Schneider H, Sarker SA, Bardhan PK, Mahalanabis D, Fuchs GJ, Gyr N (2000) Partially hydrolyzed guar gum-supplemented oral rehydration solution in the treatment of acute diarrhea in children. J Pediatr Gastroenterol Nutr 31: 503-507 
Andre T, Boni C, Mounedji-Boudiaf L, Navarro M, Tabernero J, Hickish T, Topham C, Zaninelli M, Clingan P, Bridgewater J, Tabah-Fisch I, de Gramont A, Multicenter International Study of Oxaliplatin/5-Fluorouracil/Leucovorin in the Adjuvant Treatment of Colon Cancer I (2004) Oxaliplatin, fluorouracil, and leucovorin as adjuvant treatment for colon cancer. $N$ Engl J Med 350: 2343-2351

Andre T, Colin P, Louvet C, Gamelin E, Bouche O, Achille E, Colbert N, Boaziz C, Piedbois P, Tubiana-Mathieu N, Boutan-Laroze A, Flesch M, Billiau V, Buyse M, Gramont A (2001) Randomized adjuvant study comparing two schemes of 5-fluorouracil and leucovorin in stage B2 and C colon adenocarcinoma: study design and preliminary safety results. Groupe d'Etude et de Recherche Clinique en Oncologie Radiotherapies. Semin Oncol 28: 35-40

Andre T, Colin P, Louvet C, Gamelin E, Bouche O, Achille E, Colbert N, Boaziz C, Piedbois P, Tubiana-Mathieu N, Boutan-Laroze A, Flesch M, Buyse M, de Gramont A (2003) Semimonthly versus monthly regimen of fluorouracil and leucovorin administered for 24 or 36 weeks as adjuvant therapy in stage II and III colon cancer: results of a randomized trial. J Clin Oncol 21: 2896-2903

Anonymous (1996) Randomized study on preoperative radiotherapy in rectal carcinoma. Stockholm Colorectal Cancer Study Group. Ann Surg Oncol 3: 423-430

Banasaz M, Norin E, Holma R, Midtvedt T (2002) Increased enterocyte production in gnotobiotic rats mono-associated with Lactobacillus rhamnosus GG. Appl Environ Microbiol 68: 3031-3034

Carde P, Cosset-Delaigue M, Laplanche A, Chareau I (1989) Classical external indwelling central venous catheter versus totally implanted venous access systems for chemotherapy administration: a randomized trial in 100 patients with solid tumors. Eur J Cancer Clin Oncol 25: 939-944

Cremonini F, Di Caro S, Nista EC, Bartolozzi F, Capelli G, Gasbarrini G, Gasbarrini A (2002) Meta-analysis: the effect of probiotic administration on antibiotic-associated diarrhoea. Aliment Pharmacol Ther 16: $1461-1467$

de Gramont A, Bosset JF, Milan C, Rougier P, Bouche O, Etienne PL, Morvan F, Louvet C, Guillot T, Francois E, Bedenne L (1997a) Randomized trial comparing monthly low-dose leucovorin and fluorouracil bolus with bimonthly high-dose leucovorin and fluorouracil bolus plus continuous infusion for advanced colorectal cancer: a French intergroup study. J Clin Oncol 15: 808-815

de Gramont A, Louvet C, André T (1997b) A simplified bimonthly regimen with leucovorin (LV) and 5-fluorouracil (5-FU) for metastatic colorectal cancer (MCRC). Proc Am Soc Clin Oncol 16: A1019

Delia P, Sansotta G, Donato V, Messina G, Frosina P, Pergolizzi S, De Renzis C, Famularo G (2002) Prevention of radiation-induced diarrhea with the use of VSL\#3, a new high-potency probiotic preparation. Am J Gastroenterol 97: 2150-2152

Freedman LS, White SJ (1976) On the use of Pocock and Simon's method for balancing treatment numbers over prognostic factors in the controlled clinical trial. Biometrics 32: 691-694

Galandiuk S, Wieand HS, Moertel CG, Cha SS, Fitzgibbons Jr RJ, Pemberton JH, Wolff BG (1992) Patterns of recurrence after curative resection of carcinoma of the colon and rectum. Surg Gynecol Obstet 174: 27-32

Gleeson NC, Fiorica JV, Mark JE, Pinelli DM, Hoffman MS, Roberts WS, Cavanagh D (1993) Externalized Groshong catheters and Hickman ports for central venous access in gynecologic oncology patients. Gynecol Oncol 51: $372-376$

Homann HH, Kemen M, Fuessenich C, Senkal M, Zumtobel V (1994) Reduction in diarrhea incidence by soluble fiber in patients receiving total or supplemental enteral nutrition. J Parenter Enteral Nutr 18: $486-490$

Huang JS, Bousvaros A, Lee JW, Diaz A, Davidson EJ (2002) Efficacy of probiotic use in acute diarrhea in children: a meta-analysis. Dig Dis Sci 47: $2625-2634$

Kapiteijn E, Marijnen CA, Nagtegaal ID, Putter H, Steup WH, Wiggers T, Rutten HJ, Pahlman L, Glimelius B, van Krieken JH, Leer JW, van de Velde CJ (2001) Preoperative radiotherapy combined with total mesorectal excision for resectable rectal cancer. $N$ Engl J Med 345: $638-646$

Kerr DJ (2001) A United Kingdom coordinating committee on cancer research study of adjuvant chemotherapy for colorectal cancer: preliminary results. Semin Oncol 28: $31-34$

Khaled Z, Guandalini S, Hendrickson BA (2003) Filtered supernatant of Lactobacillus GG (LGG) and VSL\#3 prevent Crohn's diasese (CD) mucosa adherent $-E$. coli -induced degradation of tight junctions (TJ) associated proteins occludin and Zo-1. I Pediatr Gastroenterol Nutr 36: 520

Kock H, Pietsch M, Krause U, Wilke H, Eigler F (1998) Implantable vascular access systems: experience in 1500 patients with totally implanted central venous port systems. World J Surg 22: 12-16

Kohne CH, Wils J, Lorenz M, Schoffski P, Voigtmann R, Bokemeyer C, Lutz M, Kleeberg C, Ridwelski K, Souchon R, El-Serafi M, Weiss U, Burkhard $\mathrm{O}$, Ruckle H, Lichnitser M, Langenbuch T, Scheithauer W, Baron B, Couvreur ML, Schmoll HJ, European Organization of R and Treatment of Cancer Gastrointestinal Group S (2003) Randomized phase III study of high-dose fluorouracil given as a weekly 24 -h infusion with or without leucovorin versus bolus fluorouracil plus leucovorin in advanced colorectal cancer: European Organization of Research and Treatment of Cancer Gastrointestinal Group Study 40952. J Clin Oncol 21: $3721-3728$

Labianca R, Marsoni S, Pancera G, Torri V, Zaniboni A, Erlichman C, Pater J, Shepherd L, Zee B, Seitz JF, Milan C, Pignon JP (1995) Efficacy of adjuvant fluorouracil and folinic acid in colon cancer. International Multicentre Pooled Analysis of Colon Cancer Trials (IMPACT) investigators. Lancet 345: 939-944

Mack DR, Ahrne S, Hyde L, Wei S, Hollingsworth MA (2003) Extracellular MUC3 mucin secretion follows adherence of Lactobacillus strains to intestinal epithelial cells in vitro. Gut 52: 827-833

Mattar AF, Drongowski RA, Coran AG, Harmon CM (2001) Effect of probiotics on enterocyte bacterial translocation in vitro. Pediatr Surg Int 17: $265-268$

Meier R, Beglinger C, Schneider H, Rowedder A, Gyr K (1993) Effect of a liquid diet with and without soluble fiber supplementation on intestinal transit and cholecystokinin release in volunteers. J Parenter Enteral Nutr 17: $231-235$

Meier R, Burri E, Steuerwald M (2003) The role of nutrition in diarrhoea syndromes. Curr Opin Clin Nutr Metab Care 6: 563-567

O'Connell M, Martenson JA, Wieand HS, Krook JE, Macdonald JS, Haller DG, Mayer RJ, Gunderson LL, Rich TA (1994) Improving adjuvant therapy for rectal cancer by combining protracted-infusion fluorouracil with radiation therapy after curative surgery. N Engl J Med 331: 502-507

O'Connell MJ, Laurie JA, Kahn M, Fitzgibbons Jr RJ, Erlichman C, Shepherd L, Moertel CG, Kocha WI, Pazdur R, Wieand HS, Rubin J, Vukov AM, Donohue JH, Krook JE, Figueredo A (1998) Prospectively randomized trial of postoperative adjuvant chemotherapy in patients with high-risk colon cancer. J Clin Oncol 16: 295-300

Ouwehand AC, Salminen S, Isolauri E (2002) Probiotics: an overview of beneficial effects. Antonie van Leeuwenhoek 82: 279-289

Parkin DM, Pisani P, Ferlay J (1999) Global cancer statistics. CA: Cancer J Clin 49(1): $33-64$

Pisani P, Parkin DM, Bray F, Ferlay J (1999) Estimates of the worldwide mortality from 25 cancers in 1990. Int J Cancer 83: 18-29

Pocock SJ, Simon R (1975) Sequential treatment assignment with balancing for prognostic factors in the controlled clinical trial. Biometrics 31: $103-115$

Saini A, Norman AR, Cunningham D, Chau I, Hill M, Tait D, Hickish T, Iveson T, Lofts F, Jodrell D, Ross PJ, Oates J (2003) Twelve weeks of protracted venous infusion of fluorouracil $(5-\mathrm{FU})$ is as effective as 6 months of bolus 5-FU and folinic acid as adjuvant treatment in colorectal cancer. Br J Cancer 88: $1859-1865$

Salminen E, Elomaa I, Minkkinen J, Vapaatalo H, Salminen S (1988) Preservation of intestinal integrity during radiotherapy using live Lactobacillus acidophilus cultures. Clin Radiol 39: 435-437

Salminen MK, Rautelin H, Tynkkynen S, Poussa T, Saxelin M, Valtonen V, Jarvinen A (2004) Lactobacillus bacteremia, clinical significance, and patient outcome, with special focus on probiotic L. Rhamnosus GG. Clin Infect Dis 38: $62-69$

Szajewska H, Kotowska M, Mrukowicz JZ, Armanska M, Mikolajczyk W (2001) Efficacy of Lactobacillus GG in prevention of nosocomial diarrhea in infants. $J$ Pediatr 138: $361-365$

Tao Y, Drabik KA, Waypa TS, Musch MW, Alverdy JC, Schneewind O, Chang EB, Petrof EO (2006) Soluble factors from Lactobacillus GG activate MAPKs and induce cytoprotective heat shock proteins in intestinal epithelial cells. Am J Physiol Cell Physiol 290: C1018-C1030

Urbancsek H, Kazar T, Mezes I, Neumann K (2001) Results of a doubleblind, randomized study to evaluate the efficacy and safety of Antibiophilus in patients with radiation-induced diarrhoea. Eur $J$ Gastroenterol Hepatol 13: $391-396$

Vaarala O (2003) Immunological effects of probiotics with special reference to lactobacilli. Clin Exp Allergy 33: 1634-1640 
Van Cutsem E, Dicato M, Wils J, Cunningham D, Diaz-Rubio E, Glimelius B, Haller D, Johnston P, Kerr D, Koehne CH, Labianca R, Minsky B, Nordlinger B, Roth A, Rougier P, Schmoll HJ (2002) Adjuvant treatment of colorectal cancer (current expert opinion derived from the Third International Conference: Perspectives in Colorectal Cancer, Dublin, 2001). Eur J Cancer 38: 1429-1436

Von Bultzingslowen I, Adlerberth I, Wold AE, Dahlen G, Jontell M (2003) Oral and intestinal microflora in 5-fluorouracil treated rats, translocation to cervical and mesenteric lymph nodes and effects of probiotic bacteria. Oral Microbiol Immunol 18: 278-284

Wolmark N, Rockette H, Mamounas E, Jones J, Wieand S, Wickerham DL, Bear HD, Atkins JN, Dimitrov NV, Glass AG, Fisher ER, Fisher B (1999) Clinical trial to assess the relative efficacy of fluorouracil and leucovorin, fluorouracil and levamisole, and fluorouracil, leucovorin, and levamisole in patients with Dukes' B and C carcinoma of the colon: results from National Surgical Adjuvant Breast and Bowel Project C-04. J Clin Oncol 17: $3553-3559$

Yan F, Cao H, Cover TL, Whitehead R, Washington MK, Polk DB (2007) Soluble proteins produced by probiotic bacteria regulate intestinal epithelial cell survival and growth. Gastroenterology 132: 562-575 